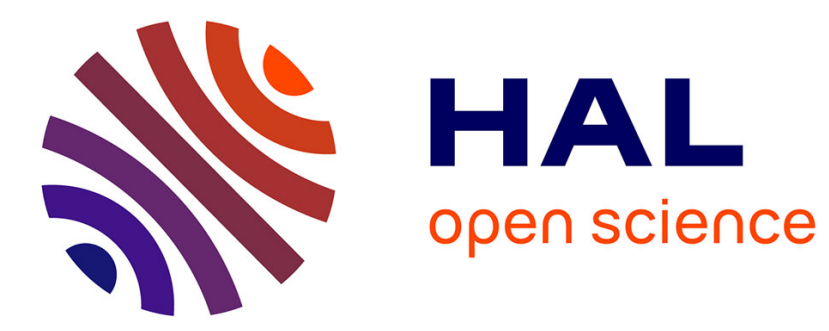

\title{
Construction sociospatiale de capabilités sexuées aux pratiques urbaines du vélo
}

David Sayagh

\section{To cite this version:}

David Sayagh. Construction sociospatiale de capabilités sexuées aux pratiques urbaines du vélo. Les Annales de la Recherche Urbaine, 2017. hal-02547574

\section{HAL Id: hal-02547574 \\ https://hal.science/hal-02547574}

Submitted on 23 Apr 2020

HAL is a multi-disciplinary open access archive for the deposit and dissemination of scientific research documents, whether they are published or not. The documents may come from teaching and research institutions in France or abroad, or from public or private research centers.
L'archive ouverte pluridisciplinaire HAL, est destinée au dépôt et à la diffusion de documents scientifiques de niveau recherche, publiés ou non, émanant des établissements d'enseignement et de recherche français ou étrangers, des laboratoires publics ou privés. 


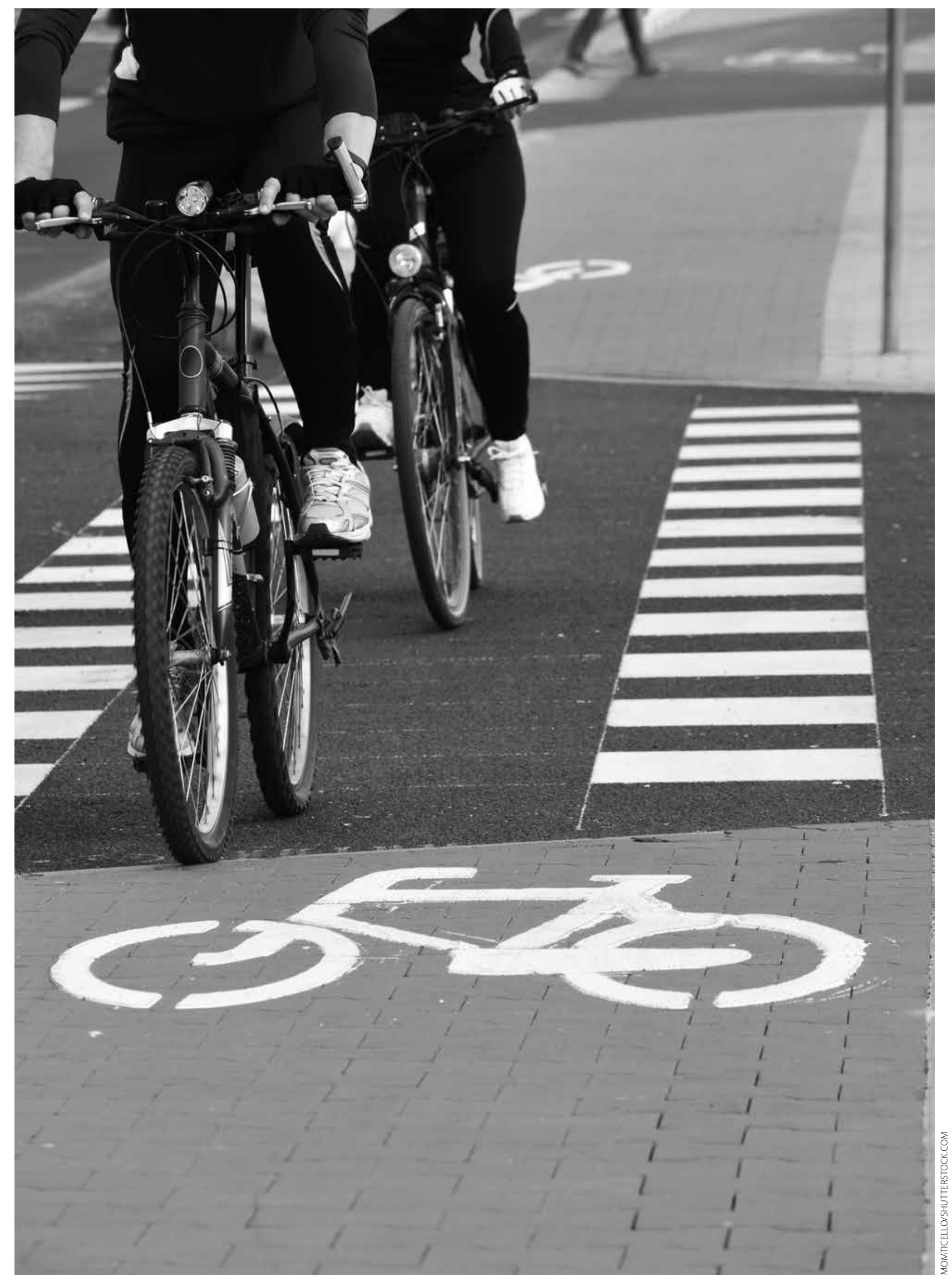




\section{Construction sociospatiale de capabilités sexuées aux pratiques urbaines du vélo}

\section{David Sayagh}

D’une manière générale, les activités physiques et sportives $^{1}$ contribuent à essentialiser l'idée de femmes physiquement faibles et d'hommes physiquement forts (Liotard et Terret, 2005). Les stéréotypes de sexe influencent les pratiques éducatives des parents, qui malgré des capacités et des demandes de soutien physique de même niveau pour les enfants des deux sexes, tolèrent mieux la prise de risque des garçons et concentrent leur effort éducatif sur l'apprentissage de l'évitement du risque chez les filles (Granié, 2008). Dans leur famille, puis à l'école, où ces dernières doivent se situer dans la cour de récréation en fonction des jeux « spatiophages » des garçons (Zaidman, 2007), les enfants apprennent en outre que certaines pratiques sont « conformes » à leur sexe, et tentent généralement de s'y associer (Vigneron, 2004). Cette socialisation sexuée est renforcée par l'influence des médias qui, en dépréciant les sportives, fréquemment jugées à partir de leur seule apparence physique, et en valorisant le sport «masculin » fondé sur la puissance, la performance et l'exacerbation de la violence, concourent à l'élaboration de la masculinité hégémonique (Fraysse et Mennesson, 2009). Par ailleurs, certains travaux, comme ceux de Raibaud (2014), pointent du doigt les aménagements publics urbains, qui tout en favorisant les pratiques urbaines des garçons, favoriseraient en contrepartie le « décrochage » des filles à partir de la classe de sixième. L'influence des pairs joue également un rôle déterminant, au point que durant l'adolescence, les filles et les garçons se défendent généralement de participer aux activités physiques qui ne correspondent pas aux valeurs de leur sexe d'appartenance ; les contrevenants n'échappant pas aux critiques et moqueries de

1. Bien que le sport ne constitue qu'un sous-ensemble de l'activité physique, spécialisé, organisé, et facilité par les organisations sportives, certains travaux convoquent encore indifféremment les deux notions, et la formule " activités physiques et sportives » reste très usitée. leurs camarades (Bramham, 2003). Durant cette période, les filles sont significativement moins " physiquement actives » que les garçons (Currie et al., 2012)2, le phénomène a récemment fait l'objet d'un chapitre dans un rapport ministériel (Naves et Octobre, 2014). Cette initiative vise essentiellement à lutter contre les stéréotypes de sexe se construisant notamment via les corps à travers la pratique d'activités physiques, débouchant sur une féminité et une masculinité «normales/idéales-typiques » participant de la cristallisation des "natures "féminine" et "masculine" » (Mennesson et Neyrand, 2010). Les pratiques du vélo ne sont pas épargnées, aussi bien lorsque celles-ci sont étudiées en tant qu'activités sportives (Naves et Octobre, 2014) ${ }^{3}$ que lorsqu'elles sont interrogées dans le champ de la mobilité (Papon, 1997). Or, en s'intéressant notamment aux modes de déplacements actifs, une étude australienne révèle que la perception de l'insécurité du quartier de résidence par les parents (risque d'accidents, d'agression verbale ou physique) est associée à une restriction de l'activité physique des adolescentes supérieure à celle établie pour les adolescents du même âge (Carver et al., 2012). À ce propos, Oppenchaim (2012) constate qu'après quatorze ans, les filles résidant

2. Les filles se déclarant systématiquement moins actives que les garçons dans les quarante et un pays sondés par la Health Behaviour in School-Aged Children Study, par rapport à une recommandation de soixante minutes d'activité physique cinq ou six jours par semaine. À onze ans, $19 \%$ des filles sont jugées suffisamment actives, contre $28 \%$ des garçons.

3. Parmi les adolescents âgés entre douze et dix-sept ans déclarant pratiquer le vélo comme une activité sportive (désignant ici « pratiques libres et en club confondues »), 61 \% sont des garçons.

Les Annales de la recherche urbaine $n^{\circ} 112,2017$, pp. 126-137, C MEEM, Puca. 
en zones urbaines sensibles $\left(\mathrm{Zus}^{4}\right)$ se voient attribuer par leurs parents un traitement spécifique, notamment caractérisé par un accompagnement des mobilités plus fréquent qu'auparavant. Des traitements différenciés que les travaux de Rivière (2014) permettent notamment d'associer aux premières transformations physiques connues par le corps des jeunes filles. C'est précisément lorsque celles-ci commencent à être dotées des « attributs corporels de la féminité » (Mardon, 2010) que leur présumée vulnérabilité permet aux parents de justifier le peu d'autonomie qui leur est alors accordée (Rivière, 2014). Ces indications sont à considérer avec attention. D'une part, parce que nous savons que le degré d'autonomie laissé aux enfants dans leurs déplacements n'est pas sans influence sur leurs dispositions futures vis-à-vis de la mobilité (Kaufmann et Flamm, 2003) ; d'autre part parce que de ces processus de socialisations différenciées émergent des dispositions sexuées ou dispositions de genre (Goffman, 2002)5 participant de la naturalisation des différences sexuées et de la reproduction de la «domination masculine » (Bourdieu, 1998).

\section{Le concept de capabilité comme outil d'analyse}

À l'instar de Lahire (2002), nous envisageons le paradigme de disposition comme une abstraction nécessaire à l'analyse, désignant la tendance, l'inclination, la propension, à agir, à penser, à être d'un acteur. Et à la manière de Darmon (2010), nous appréhendons la socialisation comme un processus d'incorporation progressif et sans cesse renouvelé de dispositions permettant à l'individu de se situer et d'interagir dans les différents mondes sociaux dans lesquels il évolue. En convoquant ces deux paradigmes, nous postulons que les individus incorporent des dispositions différenciées, ne leur permettant pas d'envisager les mêmes possibilités réelles de fonctionnement. Or, cet ensemble d'alternatives parmi lesquelles une personne peut réellement choisir constitue la définition même du paradigme de capabilité développé par Sen (1988). Selon lui, la génération d'une capabilité découle d'une conversion de ressources, elle-même déterminée par l'adéquation d'un ensemble de facteurs dits « de conversion ». Les ressources peuvent se définir comme les biens et les services dont une personne peut disposer pour agir (Robeyns, 2000), et les facteurs de conversion comme les éléments qui facilitent ou au contraire entravent l'utilisation de ces ressources. D'après Sen (1988), les ressources sont externes à la personne, les ressources internes étant appréhendées comme des facteurs de conversion personnels.

4. Les Zus ont depuis été supprimées et remplacées par les « quartiers prioritaires de la politique de la ville» (QPV) le ler janvier 2015.

5. Ensemble de schèmes de perception, de pensée et d'action propres à une catégorie de sexe, à l'origine de " comportements de genre » (genderisms) spécifiques.
Aussi, afin de mieux différencier les ressources des facteurs de conversion, nous faisons le choix de distinguer ce qui a trait à l'individu de ce qui se présente à lui. Pour ce faire, sur la base de la classification de Soulet (2009), nous distinguons les ressources pour être acteur (biens et services dont une personne peut disposer pour agir) des ressources pour agir (dispositions, habitudes, capacités, compétences $)^{6}$. Et, sur la base de la classification de Robeyns (2000), nous différencions les facteurs de conversion individuels (acquisition/dépossession involontaire de ressources), sociaux (conditions sociales d'accès), et environnementaux (infrastructures, climat...). En outre, nous désignons par " pratiques du vélo », toute pratique du vélo inscrite en milieu urbain, impliquant le corps comme principal moteur de sa propre mobilité, et se traduisant nécessairement par une activité physique.

En nous émancipant de typologies réductrices vis-à-vis des finalités de pratique (récréative, utilitaire, hygiénique, sportive.... $)^{7}$, nous nous demandons dans quelle mesure les stéréotypes de sexe contribuent à produire des capabilités sexuées vis-à-vis des pratiques urbaines du vélo au point de renforcer en retour ces stéréotypes de sexe.

\section{Méthodologie}

Nous nous appuyons pour traiter cette problématique, sur 101 entretiens semi directifs réalisés avec 42 garçons et 38 filles âgés de 17 ou 18 ans, ainsi que 26 de leurs parents ${ }^{8}$, dans des milieux géographiques et sociaux variés des métropoles de Montpellier et de Strasbourg, effectués pour environ un tiers en lycée (général, technologique, et professionnel) ${ }^{9}$, pour un tiers sur le lieu d'habitation des adolescents, et pour le dernier tiers dans quatre centres sociaux culturels de quartiers prioritaires de Strasbourg (Hautepierre, L'Aquarium-Cronenbourg, Audelà des Ponts-Port du Rhin, et Elsau). Visant en priorité à reconstituer a posteriori les parcours d'adolescents (pratiquants et non pratiquant) vis-à-vis de leurs pratiques et/ou perceptions du vélo ${ }^{10}$, ceux-ci ont parfois été accompagnés d'observations répétées aux abords des lycées et des centres socioculturels, ainsi que d'interviews informelles avec plusieurs animateurs, professeurs d'EPS, responsables d'établissement, membres salariés et bénévoles d'associations de promotion du vélo, et «Éducateurs mobilité à vélo » (EMV).

6. Soulet distingue un troisième type de ressources dites « ressources de l'action » (capitaux), ne pouvant être scrupuleusement appréhendées par le biais des entretiens biographiques réalisés.

7. Lesquelles peuvent de toute manière être cumulées par un même pratiquant, voire au cours d'une même pratique. C'est par exemple le cas d'un adolescent qui, parce qu'il trouve cela plus ludique que le bus, parce que "ça (lui) fait faire du sport », et que "ça fait les jambes », se rend au lycée à vélo en ponctuant son déplacement par une course avec un copain, deux dérapages...

8. La somme dépasse 101 dans la mesure où certains parents (père + mère) ont été interviewés simultanément (lors d'un même entretien).

9. Lycée général et technologique Jean-Monnet de Montpellier, lycée général, technologique et professionnel Jean-Rostand, et lycée général et technologique Jean-Monnet de Strasbourg.

10. La question centrale étant : « Est-ce que tu peux me raconter ta propre histoire du vélo depuis que tu as appris à en faire jusqu'à aujourd'hui? » 


\section{Des ressources pour être acteur, sexuées}

Si l'on s'intéresse aux ressources pour être acteur, soit les biens et les services dont les adolescents peuvent disposer pour agir, on ne peut passer à côté du fait que les filles et les garçons ne disposent pas des mêmes types de vélos. En effet, les cadres de type torpille, col de cygne mais aussi les cadres dits « mixtes » sont prisés par une plus grande proportion de filles, alors que les autres types de cadres (cadre en diamant ${ }^{11}$, VTT, cadre suspendu, BMX, course, demi-course...) sont adoptés dans une bien plus grande mesure par les garçons. Mais le clivage ne s'arrête pas là. Si les vélos roses, violets, verts anis et autres teintes pastel sont plus couramment utilisés par des filles, les vélos verts foncés et bruns sont adoptés par une plus grande proportion de garçons. En outre, les vélos équipés de lumière, et/ ou de garde-boue, de pare-chaîne, de porte-bagage arrière, de panier et de béquille sont davantage utilisés par des filles. Et les vélos pourvus de suspensions avant, voire de suspensions avant et arrière, ceux équipés de compteurs, ceux n'ayant qu'un seul frein, voire pas de frein (fixie), sont adoptés par une plus grande proportion de garçons.

Le fixie, «fixe » ou " pignon fixe », désigne un vélo monté avec une roue arrière dont l'unique pignon est solidaire de celle-ci. Contrairement à la roue libre, qui permet d'interrompre momentanément le pédalage, le pignon fixe contraint à pédaler sans interruption. Aussi, bien que la loi française l'interdise (cf. art. 315-3 du code de la route), un fixie véritable ne possède pas de frein, et nécessite pour être bridé d'un blocage sec et puissant du pédalier, faisant déraper le vélo : freinage technique périlleux désigné par l'anglicisme skid (dérapage). Si nombre de puristes ne jurent que par le skid ou le skip (variante du skid) sur des vélos toujours plus épurés et légers, la plupart des «pignons fixes » disposent aujourd'hui d'un frein avant à utiliser « en cas d'urgence », fixé sur un guidon raccourci pour faciliter les slaloms entre les voitures. Bien que demeurant très marginal, le mouvement n'a cessé de croître depuis ses prémisses aux États-Unis (années 1990) ${ }^{12}$, puis en Europe (milieu des années 2000), faisant du « fixe », le vélo urbain tendance, prisé quasi exclusivement par des garçons/hommes, tant dans la métropole de Montpellier que dans celle de Strasbourg.

11. Ou « cadre classique », soit le type le plus ancien et le plus courant. Il est aussi qualifié de " cadre homme ", par opposition au cadre ouvert, conçu pour être adapté aux vêtements « féminins ».

12. Bien que le pignon fixe soit à la naissance même du vélo (la roue libre n'a fait son apparition qu'en 1897), la régression technologique incarnée par ce retour aux sources constitue un phénomène récent.

\section{Des habitudes sexuées : prudence pour les filles, confrontations ludiques pour les garçons}

Le clivage ne se limite pas aux supports et équipements. Outre le fait que les adolescentes soient beaucoup moins nombreuses à pratiquer le vélo que leurs homologues masculins, celles-ci font état d'habitudes d'actions différenciées. Davantage que les garçons, elles ont tendance à « tracer» $\left(\right.$ Zaffran, 2016) ${ }^{13}$. En proportion, elles sont en outre plus nombreuses à pratiquer exclusivement accompagnées, uniquement en journée, à éviter à tout prix la cohabitation vélo-voitures, voire à pratiquer quasi exclusivement sur des pistes cyclables en site propre. Aussi, plus fréquemment que les garçons, elles adoptent une position " dos droit ", descendent automatiquement de leur vélo pour traverser une rue ou franchir un trottoir, ou encore, attendent systématiquement que le feu soit vert pour traverser.

À l'inverse, les garçons ont dans une plus large mesure que les filles tendance à rouler en cohabitation avec les voitures, ou sur la plateforme du tramway ${ }^{14}$. En proportion, ils sont beaucoup plus nombreux à tourner en rond, à faire la course (entre " cyclistes ", mais aussi contre des bus, tramways et voitures), à rouler la nuit et/ ou sous la pluie. Davantage que les filles, ils traversent au feu rouge, franchisent des équipements urbains (trottoirs, escaliers...) ou des obstacles délibérément bricolés à partir de matériaux de construction de travaux publics sans poser pied à terre, et réalisent des figures de toutes sortes (déraper, rouler en marche arrière, sauter, cabrer ou lâcher le guidon). Les adeptes du fixie s'adonnent à des pratiques diverses et variées, comme l'alleycat (course cycliste urbaine comportant des points de passage obligatoires), le ride (sortie durant laquelle sont recherchées par alternance, performances de vitesse, dérapages et trackstand maîtrisés), le street $^{15}$, ou encore le Hardcourt bike polo (« polo à vélo sur terrain dur »), se pratiquant la plupart du temps sur le bitume, produisant des percussions et des chutes particulièrement violentes. Autant de pratiques exigeant des techniques particulières peu accessibles pour qui n'est pas enclin à prendre des risques délibérés et/ou à se faire mal pour apprendre, une disposition qui s'avère fortement sexuée chez les adolescents interrogés.

13. Zaffran (2016) utilise le terme pour désigner le fait que les filles préfèrent «se déplacer d'un lieu à un autre, et ne restent pas longtemps au même endroit ».

14. Circulation interdite en France sur les plates-formes non situées en « site banal».

15. Le track-stand est une technique visant à rester à l'arrêt sans poser les pieds au sol tout en réalisant des petits mouvements d'avant en arrière, tandis que le barspin consiste à faire tourner son guidon à $360^{\circ}$ et à le rattraper après son tour complet, figure qui nécessite l'absence de frein à l'avant. 


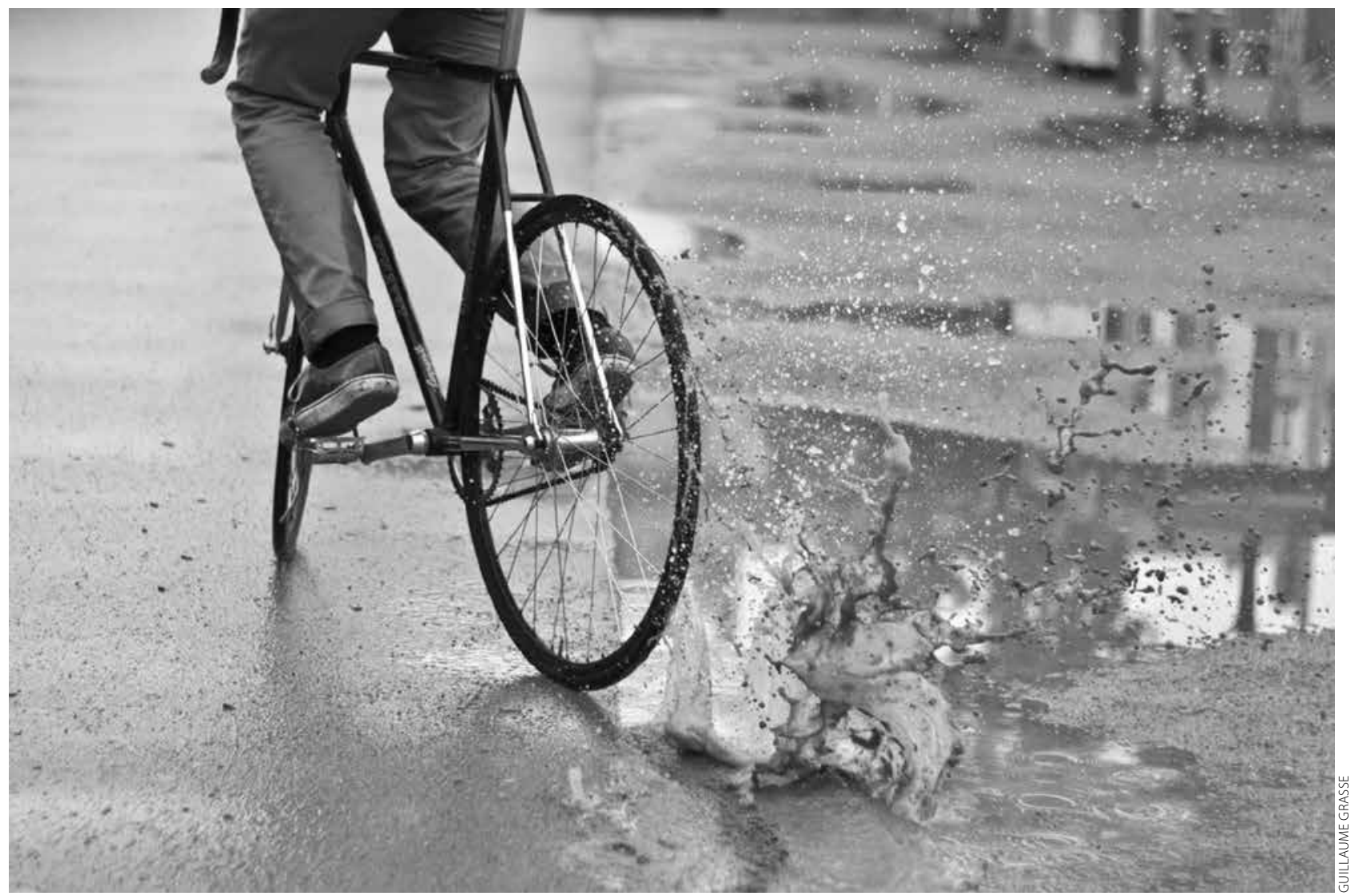

\section{Des dispositions sexuées}

L'inclination à faire du vélo « à condition que ça ne fasse pas mal » se révèle même être la disposition « féminine ${ }^{16}$ » la plus prégnante. Aussi bien observée chez celles qui déclarent aimer faire du vélo, elle est également présentée par les mères de familles, par ailleurs plus nombreuses que les pères à déclarer envisager l'achat d'un vélo électrique :

J'envisage d'acheter un vélo électrique, pour que justement, euh. . quand en fait ça devient dur euh... ben voilà j'enclenche l'électrique et puis comme ça c'est déjà moins dur et voilà y a plus que le plaisir qui reste quoi (Nathalie, cinquante ans, Saint-Drézéry).

Or, dans une plus grande proportion que les garçons, les filles sont disposées à penser le vélo comme un loisir récréatif collectif ( promenades » en famille ou entre amies), fortement associé aux beaux jours, sans quoi il ne s'agirait plus d'un loisir : "On en fait l'été, le vélo quand ça fait, euh froid, quand il pleut ou quoi, c'est pffffuu, c'est pas coo [cool] c'est pas détente quoi ! » (Lucie, dix-sept ans, Montpellier centre). Ainsi, davantage que les garçons, les filles sont disposées à redouter, voire « détester » les

16. Nous entendons par « dispositions féminines » des dispositions socialement construites désignant des inclinations observées chez une proportion notablement plus grande de femmes (Zolesio, 2010). montées, et plus généralement, à penser le vélo comme une activité «fatigante». Aussi, certaines adolescentes estiment que pour « être stylée » à vélo, une fille doit avoir une selle basse, et se tenir « le dos bien droit », une disposition à penser jamais manifestée par des garçons.

En outre, l'analyse des discours permet de relever que certaines habitudes communes, comme le fait de ne pas porter de casque, ou encore de rouler sur le trottoir (habitudes observées tant chez les filles que chez les garçons), ne sont pas moins révélatrices de dispositions sexuées que la plupart des habitudes sexuées. En effet, si dans une plus grande mesure, les filles justifient le « non-port du casque » en dénonçant ses propriétés «inesthétiques ", les garçons évoquent son inutilité dans une plus grande proportion : "Je me suis jamais dit "il faut mettre un casque, ça va me sauver la vie si je tombe" » (Lionel, dixhuit ans, Saint-Drézéry). De même, si les filles justifient fréquemment le fait de rouler sur le trottoir par leur « peur des voitures », les garçons, pourtant également disposés à craindre le trafic, affirment couramment recourir à cette option pour «aller plus vite».

D'ailleurs, alors que la quasi-totalité des garçons interrogés n'associent à la pratique du vélo d'autres dangers que celui que représente la voiture (ou le trafic en général), et déclarent systématiquement se sentir plus " en sécurité » à pied qu'à vélo, près d'un tiers des filles atteste du sentiment inverse, avançant l'idée qu'à vélo « tu peux partir 
plus vite en cas de danger ». Une disposition à penser le vélo animée par une peur sexuée (Lieber, 2008) incarnée par l'éventualité d'une agression sexuelle (physique ou verbale). Aussi, nous relevons que la peur de pratiquer la nuit, disposition "féminine " par excellence, s'avère tant prégnante chez les filles déclarant avoir été témoin ou victime d'agression, ou de tentatives d'agression (physique ou verbale) que chez celles affirmant n'avoir jamais vécu la moindre "expérience déplaisante » dans l'espace urbain et/ou dans les transports en commun. En outre, alors que cette peur est déclarée de manière plus systématique chez les filles non pratiquantes, ou ayant rarement pratiqué le vélo, et/ou chez celles qui sortent peu voire jamais après les cours, la disposition à se sentir plus « en sécurité " à vélo qu'à pied (jour ou nuit confondus) est, de manière très significative, formulée par les pratiquantes les plus assidues ${ }^{17}$ de notre échantillon, qui sont aussi les plus enclines à sortir de jour comme de nuit.

De manière générale, les filles font état d'inclinaisons à penser leur vulnérabilité relativement similaires à celles présentées par leurs mères. C'est le cas de la disposition à craindre la pratique de nuit, mais aussi à penser le vélo comme un mode de déplacement rassurant, permettant de limiter les risques d'agression, ou d'en échapper plus rapidement. Lorsqu'elle est manifestée, cette dernière disposition est en effet partagée de manière quasi systématique par les mères, qui affirment par ailleurs avoir elles-mêmes appris à faire du vélo à leur fille (alors que ce rôle est généralement assuré par le père) et déclarent très explicitement vouloir encourager l'autonomie et/ou l'indépendance de leur fille en leur accordant un maximum de liberté. Une volonté se traduisant pour les filles par un champ spatio-temporel du possible plus large que leurs camarades (moindres restrictions de sortie vis-à-vis des horaires et des limites territoriales à ne pas dépasser).

Malgré les différences observées chez les filles, les garçons sont disposés, dans une plus grande mesure, à ne pas craindre la pratique du vélo seul et/ou de nuit, à envisager la pratique du vélo comme principal moyen de déplacement, mais aussi en tant que sport (fédération, club...) ou loisir sportif (balades/randonnées), de manière acrobatique (à la recherche de prouesses techniques), ou encore en tant que mode de préparation physique ${ }^{18}$.

\section{Des compétences sexuées}

Parce que les compétences requises peuvent fortement différer selon l'environnement et le type de pratique (cf. l'exemple du fixie), le choix d'appréhender le large

17. Celles qui déclarent pratiquer plusieurs fois par semaine.

18. C'est notamment le cas des garçons pratiquant un sport en compétition, qui déclarent faire du vélo pour « garder la forme » pendant les périodes de vacances. champ des pratiques urbaines du vélo implique, pour faciliter l'analyse, d'identifier des indicateurs de compétences élémentaires mobilisées dans la plupart de ces pratiques. Pour ce faire, nous avons contacté la totalité des véloécoles de la Fédération française des usagers de la bicyclette, puis synthétisé une série de critères et d'indicateurs communs aux grilles utilisées par la plupart d'entre elles pour évaluer les niveaux de compétence et de progression de leurs élèves.

\begin{tabular}{|l|l|}
\hline \multicolumn{1}{|c|}{ Critères } & \multicolumn{1}{c|}{ Indicateurs } \\
\hline \multirow{4}{*}{ Équilibre } & Savoir prendre un virage serré sans poser le pied à terre \\
\cline { 2 - 2 } & Lâcher une main, bras tendu en ligne droite \\
\cline { 2 - 2 } Efficience & Lâcher une main, bras tendu dans un virage \\
\hline \multirow{4}{*}{$\begin{array}{l}\text { Adaptabilité au } \\
\text { terrain }\end{array}$} & Savoir changer les vitesses \\
\cline { 2 - 2 } & Changer les vitesses sans regarder ses mains \\
\cline { 2 - 2 } & Savoir rouler sur une surface non lisse \\
\cline { 2 - 2 } & Savoir descendre une forte pente \\
\hline \multirow{4}{*}{$\begin{array}{l}\text { Mécanique de } \\
\text { base }\end{array}$} & Entretien courant \\
\cline { 2 - 2 } & Crevaison/réparation \\
\cline { 2 - 2 } & Équipement de sécurité \\
\cline { 2 - 2 } & Régler les freins \\
\hline
\end{tabular}

Le caractère rudimentaire des indicateurs retenus permet de faire parler la grille. En effet, confrontée aux données issues des entretiens, celle-ci nous permet d'affirmer que parmi les individus déclarant ne pas disposer de ces compétences mobilisables dans la plupart des pratiques urbaines du vélo, on retrouve une grande majorité de filles. Certaines, qui ont peu pratiqué, déclarent devoir poser le pied à terre avant de prendre un virage serré. D'autres, qui n'ont pas pratiqué depuis leur entrée au collège, déclarent même ne pas savoir si elles seraient capables d'en faire aujourd'hui (à dix-sept ou dix-huit ans). Toutefois, nous notons que les filles de notre échantillon présentant une tendance à se penser moins vulnérables à vélo qu'à pied, déclarent toutes maitriser ces compétences, à l'exception des compétences relatives à la " mécanique de base ", s'avérant de loin être l'axe le plus clivé. En effet, à titre d'exemple, quand la majorité des garçons déclarent savoir réparer une roue de vélo crevée, c'est le cas de près d'une adolescente sur dix, lesquelles font d'ailleurs partie de celles étant disposées à se penser moins "en sécurité » à vélo qu'à pied. Mais si la totalité des garçons déclarant détenir cette compétence mécanique affirment que celleci leur a été transmise par leur père, ce n'est le cas pour aucune des filles concernées, lesquelles déclarent une transmission par leur mère, une tante, ou un organisme de promotion du vélo. Mais les pratiques éducatives différenciées vis-à-vis des pratiques du vélo ne se limitent pas aux aspects mécaniques du vélo. 


\section{Des facteurs de conversion sociaux sexués}

En effet, bien que selon leurs déclarations (et celles de leurs parents), les garçons et les filles apprennent à faire du vélo sensiblement au même âge, ceux-ci bénéficient au cours de leurs parcours des traitements différenciés, conférant généralement aux filles un ensemble d'alternatives de fonctionnements plus restreint. Quatre facteurs apparaissent particulièrement significatifs à ce titre : dans une plus grande mesure que les garçons, les filles 1) apprennent à faire du vélo dans un espace relativement exigu, explicitement délimité, voire cloisonné ${ }^{19}$; 2) se voient formellement interdire le droit de sortir du quartier de résidence/du village ; 3) se voient formellement interdire le droit de pratiquer seules à partir du collège ; et enfin 4) se voient formellement interdire le droit de pratiquer la nuit ${ }^{20}$.

Il apparaît fréquemment que ces interdictions n'aient pas besoin d'être formulées. Ainsi, à propos de l'idée de sortir du village pendant ses années collège, Annabelle (dix-huit ans, Restinclières) déclare par exemple : " Je crois pas qu'ils me l'aient vraiment interdit, mais t'façon, ça me serait pas venu à l'idée, j'en voyais pas vraiment l'intérêt. »Un constat qui dénote un fort ancrage de la disposition à penser qu'il n'est tout simplement " pas normal pour une jeune fille» de s'aventurer seule, hors de l'espace connu ${ }^{21}$.

En outre, il nous paraît important de noter que si durant l'école primaire, les garçons comme les filles sont susceptibles de se rendre à l'école seul.e.s, notamment dans les villages, couramment à partir du CM2, les filles pratiquant seules à partir des années collèges sont beaucoup plus rares. État de fait s'accompagnant du constat que les adolescents déclarant une pratique du vélo « contrainte ${ }^{22}$ », sont dans une plus grande proportion des garçons, les filles se faisant en général plus fréquemment accompagner par leurs parents. Enfin, il ressort de nos entretiens que si les capabilités sexuées relevées semblent concerner tous les milieux urbains, celles-ci s'avèrent plus prégnantes dans les quartiers prioritaires.

\section{Des capabilités sexuées renforcées en quartier prioritaire}

En effet, nous remarquons d'abord que si les adolescents de ces quartiers déclarent plus fréquemment que

19. Exemple : un jardin ou une cour fermé(e), voire une terrasse ou un balcon.

20. Parfois même lorsqu'elles sont majeures mais résident chez leurs parents.

21. Disposition partagée par plusieurs mères, pères, et garçons de notre échantillon.

22. Ils/elles préfèreraient une autre option. la moyenne ne pas avoir disposé ou ne pas disposer d'un vélo personnel, cela concerne dans une bien plus grande mesure les filles. Aussi, parmi les adolescentes déclarant ne pas maîtriser les compétences élémentaires communes aux pratiques urbaines du vélo et/ou assurant ne jamais avoir pratiqué la nuit, et/ou seules, et/ou en cohabitation avec les voitures, on retrouve une grande majorité de filles résidant en quartier prioritaire, dont les mères n'ont pour la plupart jamais appris à faire du vélo. Dans une plus large mesure que les autres adolescentes, les filles résidant dans ces quartiers font état de dispositions à craindre la nuit, une peur souvent fondée sur l'imaginaire d'un univers effrayant parce que méconnu, dans lequel évoluent essentiellement des personnes mal intentionnées, notamment incarnés par les prostituées et les buveurs d'alcool. Un schéma représentatif explicité par les propos tenus par Nacera (dix-sept ans, Port du Rhin, Strasbourg) pour justifier le fait de n'avoir jamais fait de vélo de nuit:

Non la nuit, il fait noir... 'fin il fait sombre, on voit pas, c'est dangereux, ça fait peur [...]. Dans le quartier, il y a des filles qui se promènent la nuit, voilà quoi, et euh... y a aussi des fous 'fin, qui boivent et tout ça.

En outre, la pratique du vélo par une fille à partir de la préadolescence semble aller à l'encontre des normes sociales en vigueur dans ces environnements. Une tendance tant observable dans les quartiers prioritaires de Montpellier que dans ceux de Strasbourg, que le cas d'Amina (dix-sept ans, Hauts de Massane, Montpellier) permet d'illustrer. Après avoir partagé un vélo avec sa sœur jusqu'à ses huit ans, Amina se voit offrir « un grand vélo » par son père. Un vélo qu'elle n'utilisera qu'une fois puisque, suite à une chute lors de sa première utilisation, elle déclare ne plus jamais en avoir fait en France. Une mention essentielle puisqu'elle affirme en parallèle en faire depuis plusieurs années tous les étés « pour le loisir » et pour ses déplacements avec ses cousines, dans le village où réside une partie de sa famille au Maroc :

En fait au Maroc je vois tout le monde en faire et tout ça 'fin, les jeunes filles enfin et tout ça, alors qu'ici je... 'fin c'est juste des garçons, enfin après j'ai pas trop envie d'en faire.

En dépit de la permanence de son appétence pour la pratique du vélo et malgré la maîtrise de compétences lui permettant de pratiquer au Maroc, les conditions sociales d'accès incarnées par les interdits sociaux à l'œuvre dans son quartier de résidence ne lui permettent pas de convertir ses ressources en alternatives réelles de fonctionnements.

Si les normes sociales s'avèrent déterminantes vis-àvis des capabilités des filles, les faibles ressources économiques des ménages de ces quartiers semblent également fortement influencer les équipements utilisés par les garçons qui, dans une bien plus grande mesure que la moyenne, sont souvent amenés à partager leur vélo. En outre, nous relevons que les fixies, qu'ils soient de type 


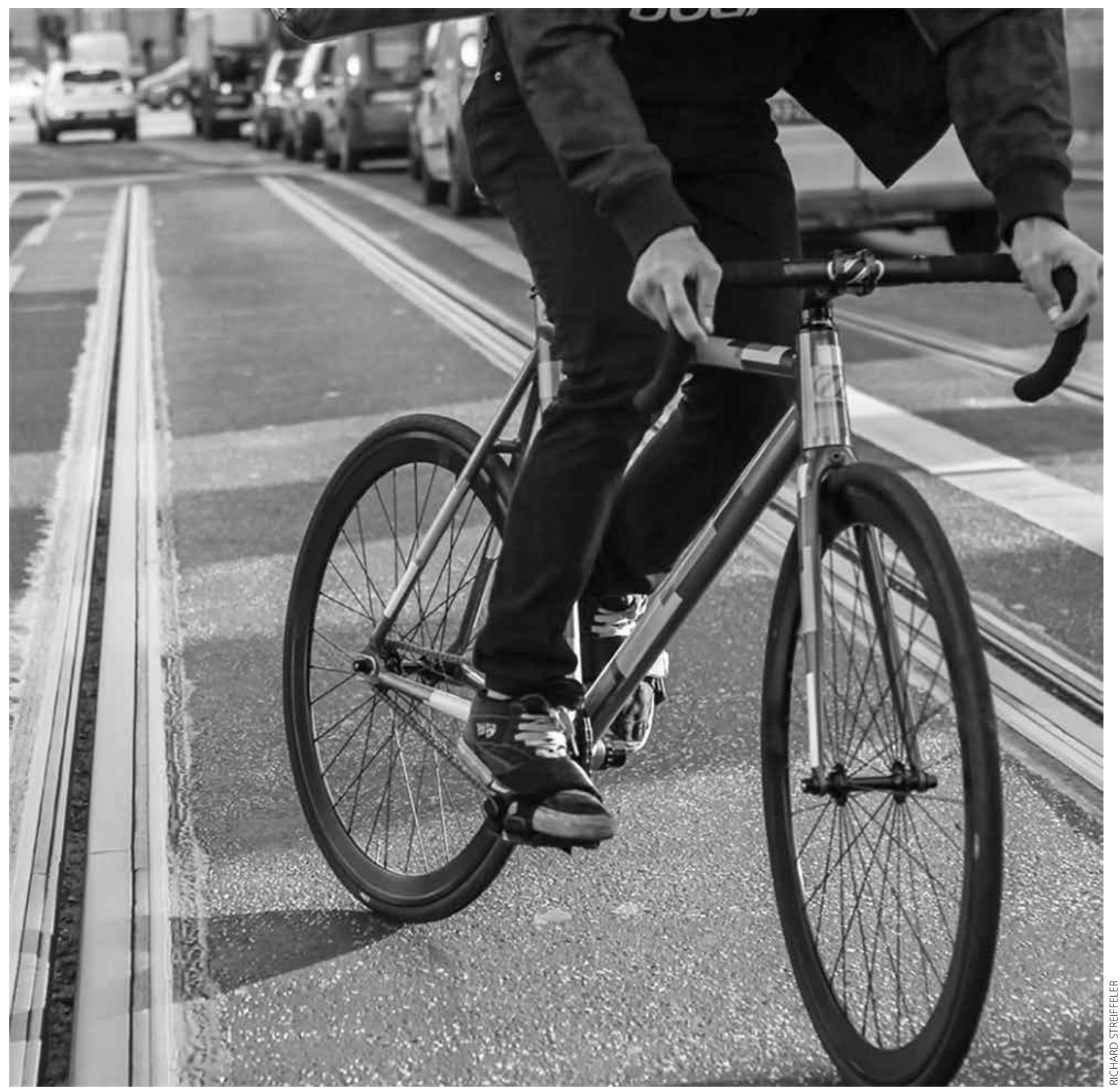

« coursier », « piste », « street », ou « polo » ${ }^{23}$, qu'ils soient neufs ou bricolés à partir d'un cadre vintage, ne sont pas présents dans ces milieux. Néanmoins, outre les espaces dédiés (ex : skate park, piste de cyclo-cross), c'est dans ces quartiers que nous avons observé le plus fréquemment des garçons se défier et s'afficher en s'adonnant à des pratiques

23. Le type « coursier » est souvent issu d'une conversion de cadre de course très compact pour faciliter le slalom dans la circulation urbaine. Le cadre du fixie "piste » reprend les codes des vélos de cyclisme de piste, particulièrement légers, et aérodynamiques. Le «street » est souvent moderne et suffisamment robuste pour réaliser certaines figures de flat (freestyle en milieu urbain). Enfin le type " polo » est généralement compact et très maniable, notamment pour faciliter la tenue en équilibre. «acrobatiques » mettant en scène des habiletés techniques et/ou valorisant la prise de risque délibérée. La pratique la plus couramment observée est le « cabrage », «lever », « roue arrière », ou encore « wheeling ». Consistant à rouler uniquement sur la roue arrière, cette figure ${ }^{24}$ est si fréquemment employée qu'on pourrait se demander s'il est mal perçu pour un jeune garçon de ces quartiers, de rouler sur deux roues. Si elle peut sembler «sauvage » (Carroux, 1978) au regard du néophyte, la figure est implicitement codifiée, et d'autant plus valorisée que la distance parcourue (sans poser la roue avant) est importante et/ou si elle est réalisée avec une seule main sur le guidon.

24. Aussi bien réalisée par les conducteurs de scooter. 


\section{Une vulnérabilité " féminine " essentialisée par une socialisation sexuée à investir l'espace}

Comme nous l'avons relevé, supposées naturellement vulnérables, les filles sont socialisées dès leur plus jeune âge à éviter les prises de risque physique et à investir des espaces restreints et cloisonnés. Durant l'adolescence, elles utilisent dans une plus large mesure que les garçons des vélos et des équipements permettant d'envisager un champ de pratiques relativement réduit, limitant notamment les manœuvres « mouvementées » et/ou à haut niveau d'habileté technique, mais favorisant les déplacements apaisés, les balades sur terrain non accidenté. À l'inverse, les supports davantage utilisés par les garçons facilitent les pratiques " acrobatiques ", et/ou de vitesse, et/ou illicites (avec un fixie par exemple), valorisant la prise de risques, la recherche de performance, l'endurance, la démonstration, la propulsion d'objets, soit autant d'éléments caractéristiques des activités physiques et sportives majoritairement appropriées par les individus de sexe masculin (Vigneron, 2004). Ainsi, les filles sont en proportion plus enclines que les garçons à pratiquer le vélo quasi exclusivement sur piste cyclable en site propre, une attitude qu'on retrouve pour les femmes dans leur ensemble, qui, d'une manière générale, privilégient les voies offrant un maximum de séparation du reste du trafic (Garrard et al., 2008). Une préférence off-road partagée par les cyclistes femmes dites « expérimentées » (Emond, 2009), et qui selon certains auteurs, devrait encourager la construction massive de pistes cyclables afin de promouvoir le vélo « féminin » (Garrard et al., 2008). Mais comment légitimer la construction de pistes cyclables sous prétexte que les femmes s'y sentent plus à l'aise sans essentialiser leurs dispositions à se sentir vulnérables dans l'espace public ? D'ailleurs, les auteurs et autres militants pointant du doigt les aménagements publics urbains, qui dans leur ensemble favoriseraient les pratiques urbaines des garçons (Raibaud, 2014), devraient selon nous se demander s'il n'est pas antinomique de vouloir lutter contre la sexuation de l'espace public sans chercher à lutter contre la sexuation des pratiques s'y inscrivant. Outre le constat global d'équipements sexués associés à des habitudes d'actions sexuées, la relation entre équipements et pratiques dévoile néanmoins certains paradoxes. En effet, on relève par exemple qu'alors que les vélos utilisés par les filles sont plus fréquemment équipés de garde-boue et de lumière(s), les garçons sont plus enclins à pratiquer sous la pluie d'une part et de nuit d'autre part. Bien qu'il puisse sembler paradoxal, ce constat semble d'abord dénoter une conformité des équipements des filles avec la disposition « féminine » incarnée par « l'attrait de la propreté » (Jodelet, 2007). Il conforte en outre la thèse de la construction sociale d'une peur sexuée prenant particulièrement sens la nuit (Lieber, 2008).

\section{Le vélo, un remède « libérateur d'accès » à la ville, réservé aux pratiquantes assidues}

Les pratiques éducatives des mères semblent jouer un rôle de transmission déterminant à cet effet, au point que leurs inclinations à penser la vulnérabilité des filles/ femmes dans l'espace public, soient souvent semblables à celles manifestées par leurs filles. Les interrelations entre ces pratiques éducatives et ces dispositions sexuées confèrent à ces dernières un champ spatio-temporel du possible plus limité que celui des garçons, les conduisant notamment à conserver leur déficit en compétences élémentaires mobilisées dans la plupart des pratiques urbaines du vélo. Si c'est d'autant plus le cas pour les compétences ayant trait à la mécanique, cette tendance semble par exemple conforter la sexuation prononcée du phénomène fixie, fortement associé à l'essor du réemploi de produits vintage, impliquant des appétences et des compétences spécifiques en matière de mécanique et de bricolage.

Néanmoins, le déficit déclaré en compétences sommaires concerne dans une moindre mesure les filles de notre échantillon, qui présentent une disposition à se penser moins vulnérables à vélo qu'à pied. Disposition largement partagée par leurs mères, qui d'une part, affirment s'être personnellement chargées de leur apprentissage du vélo, et d'autre part, encouragent délibérément leurs " automobilités ${ }^{25}$ " (Massot et Zaffran, 2007) régulières en les faisant bénéficier d'un champ spatio-temporel du possible plus large que celui observé pour la majorité des filles. En rassurant tant les pratiquantes les plus assidues que leurs mères, le vélo agit contre leur sentiment d'insécurité comme un remède " libérateur d'accès » à la ville. Analyse face à laquelle il serait tentant d'axer la promotion du vélo des filles en encourageant une transmission par les mères. Cependant, comme nous l'avons relevé, la sexuation des capabilités relatives aux pratiques urbaines du vélo ne semble jamais aussi prononcée que dans les quartiers prioritaires, dans lesquels nombre de mères ne savent pas faire de vélo. Ainsi, nous ne saurions qu'encourager le travail considérable des vélos-écoles pour adultes qui accueillent essentiellement des femmes originaires d'Afrique subsaharienne et du Maghreb, où la « culture vélo » est parfois inexistante ou souvent réservée aux hommes, au point d'être parfois considérée comme incorrecte pour une femme (Deschamps, 2013).

25. L'automobilité se définit à l'adolescence par le fait de réaliser ses déplacements seul, c'est-à-dire sans la présence d'adultes et notamment de ses parents. 


\section{Le vélo, support d'une domination masculine de l'espace public par le corps}

Il n'en demeure pas moins que dans les quartiers prioritaires plus qu'ailleurs, la voirie constitue un terrain de jeu malvenu pour les filles/femmes, où les adolescents (garçons), « traînent », « tournent », marquent leur place, affirment leur « droit de présence » (Ripoll, 2006) ou « droit de cité » par le corps (Danic, 2016), et finalement, renforcent les normes habitantes d'appropriation masculine des espaces publics. Or, comme l'explique Clair (2008), ceuxci s'accordent sur l'idée que les filles doivent faire preuve de réserve, de discrétion, leur visibilité dans l'espace public ne pouvant être légitimée que par des « visées sexuelles ». Pour protéger leur image sociale (éviter les rumeurs sur leurs vertus amoureuse et/ou sexuelles) et se conformer aux normes qu'alimentent les croyances dans l'« ordre du genre ", celles-ci chercheraient à être le moins possible seules et le plus possible invisibles à l'extérieur. Bien que pouvant sembler caricaturale, cette analyse permet de comprendre la force du facteur de conversion qu'incarne la réputation, tant celle-ci peut, de par la forte densité et l'interconnaissance entre les habitants de ces quartiers (Oppenchaim, 2012), rapidement engager celle de leur famille ou devenir le prétexte d'agressions verbales et/ou physiques (Clair, 2008). En outre, un exemple aussi extrême qu'explicite permet d'illustrer le rôle déterminant que peuvent jouer la culture et la religion vis-à-vis des capabilités liées aux pratiques du vélo : aux Pays-Bas, où le vélo est pourtant roi, une fatwa décrétée en 2007 interdit aux femmes musulmanes de pratiquer le vélo car, selon les religieux qui la répandent, « enjamber la selle du vélo suscite chez la femme une excitation sexuelle et le vélo devient, de ce point de vue, un objet prohibé » (Kleinsorge et al., 2005). Bien que n'émanant pas de l'islam le moins radical, cet exemple a selon nous le mérite de mettre en exergue le rapport complexe entre l'incorporation de dispositions favorables à la pratique du vélo et la non-disposition de son propre corps. Une problématique sensible, qui pour être abordée, conduirait à dépasser une des principales limites de notre travail, à savoir qu'ainsi que nous avons pu le constater sur nos terrains, les mères et les filles présentant les capabilités les plus limitées vis-à-vis des pratiques urbaines du vélo sont aussi les moins susceptibles de se porter volontaires pour se livrer à un entretien sur le thème du vélo.

Par ailleurs, nous avons notamment fait remarquer que les filles ne sont pas les seules à être peu susceptibles de pratiquer le fixie, également absent chez les garçons résidant en quartier prioritaire. Ce constat peut se comprendre dans la mesure où malgré les prémices d'une démocratisation des prix, la commercialisation du fixie et/ou des pièces nécessaires à sa fabrication reste l'apanage de petits commerces spécialisés implantés dans des quartiers récemment gentrifiés. En somme, le vélo, et plus particulièrement le fixie, mériteraient selon nous d'être étudiés en tant qu'instruments de distinction à la fois sexuée et sociale (Gaboriau, 1991), mais aussi spatiale, de par la « prise de pouvoir symbolique sur la ville » qu'ils semblent incarner (Clerval, 2005). D'une manière plus générale, nos résultats dénotent selon nous l'intérêt de se pencher davantage sur les dimensions « intersectionnelles » des rapports sociaux (Crenshaw, $2005^{26}$ ). Notamment en recourant au paradigme de « rapport sociaux de sexe », lequel permet d'une part de centrer l'analyse sur l'articulation des rapports sociaux (de sexes, de classe et même de race) qui s'inscrivent dans et se reproduisent par les corps, et d'autre part de penser le changement en fonction du contexte spatiotemporel (Pfefferkorn, 2007). Enfin, en cherchant à analyser les processus d'incorporation de « dispositions sexuées inversées » (Mennesson et Neyrand, 2010), il serait selon nous fructueux de se demander dans quelle mesure les cheminements des (rares) filles sachant squidder ${ }^{27}$ présentent des analogies.
26. La notion d'« intersectionnalité » propose de penser l'entrecroisement des caractéristiques sociales.

27. Francisation en référence au skid défini en début d'article. 


\section{Références bibliographiques}

Bramham P., (2003), "Boys, masculinities and PE », Sport, education and society, $n^{\circ}$ 8, pp. 57-71.

Bourdieu P., (1998), La domination masculine, Paris, Seuil.

Carroux J., (1978), «Figures urbaines du quotidien le skate sauvage », Esprit, vol. 10, pp. 26-35.

Carver A., Timperio A., Hesketh K., Crawford D., (2012), « How does perceived risk mediate associations between perceived safety and parental restriction of adolescents' physical activity in their neighborhood? », Int J BehavNutr Phys Act, $n^{\circ}$ 9, pp. 1-57.

Clair I., (2008), Les jeunes et l'amour dans les cités, Paris, Armand Colin.

Clerval A., (2005), « David Brooks, 2000, Les Bobos, Les bourgeois bohèmes », trad. par M. Thirioux et A. Nabet, Paris, Florent Massot, coll. Le livre de poche 314 p., Cybergeo : European Journal of Geography, [en ligne] http://cybergeo.revues.org/766

Crenshaw K., (2005), «Cartographie des marges: intersectionnalité, politiques de l'identité et violences contre les femmes de couleur », Les Cahiers du genre, $n^{\circ} 39$, pp. 51-82.

Currie C., Zanotti C., Morgan A. et al., (2012), « Social determinants of health and well-being among young people: Health Behaviour in School-Aged Children (HBSC) study: international report from the 2009/2010 survey », Health Policy for Children and Adolescents, $n^{\circ} 6$.

Danic I., (2016), « Les places des adolescent.e.s en zone urbaine sensible, entre attributions, approbation et retrait », Les Annales de la recherche urbaine, $n^{\circ} 111$, pp. 78-89.

Darmon M., (2010), La socialisation, Paris, Armand Colin.

Deschamps E., (2013)، «Le vélo, un coup de pouce pour les femmes immigrées à Strasbourg », La Croix [en ligne] http://www.la-croix.com/Solidarite/EnFrance/Le-velo-un-coup-de-pouce-pour-les-femmesimmigrees-a-Strasbourg-2013-10-21-1047704.
Emond C., (2009), « Explaining Gender Difference in Bicycling Behavior », Committee on Women's Issues in Transportation: ABE70.

Fraisse M., Mennesson C., (2009), « Masculinités hégémoniques et féminités : les modèles de genre dans une revue de VTT », Sciences sociales et sport, $n^{\circ} 2$, pp. 25-53.

Gaboriau P., (1991), «Les trois âges du vélo en France », Vingtième Siècle, revue d'histoire, $n^{\circ} 29$ pp. 17-34.

Garrard J., Rose G., Lo S.-K., (2008), « Promoting Transportation Cycling for Women: The Role of Bicycle Infrastructure », Preventive Medicine, ${ }^{\circ} 46$, pp. 55-59.

Goffman E., (2002), L'arrangement des sexes, Paris, La Dispute.

Granié M.-A., (2008), « Socialisation différenciée au risque : influence de l'identité sexuée sur le rapport au risque et à la règle », in M.-A. Granié, Genre, Risques, Éducation, Socialisation (GENRES). La psychologie du développement au service de la compréhension de la différence des sexes dans l'accidentologie routière. Rapport final sur subvention PREDIT/DSCR, Salon de Provence, Inrets, pp. 13-63.

Inserm Expertise collective, (2008), Activité physique. Contextes et effets sur la santé, Paris, Inserm.

Jodelet D., (2007), « Imaginaires érotiques de l'hygiène féminine intime. Approche anthropologique », Connexions, n 87, pp. 105-127.

Kaufmann V., Flamm M., (2003), Famille, temps et mobilité. État de l'art et tour d'horizon des innovations, Paris, Rapport de recherche Cnaf et Ville en mouvement.

Kleinsorge T., Affholder S., Devaux J., Parent, (2005), Committee on Equal Opportunities for Women and Men, Doc 10705, n³ 3145, 7 octobre, [en ligne] http:// assembly.coe.int/nw/Xml/XRef/Xref-XML2HTML-en. asp?fileid=11864\&lang=en

Lahire B., (2002), Portraits sociologiques. Dispositions et variations individuelles, Paris, Nathan.
Lieber M., (2008), Genre, violences et espaces publics. La vulnérabilité des femmes en question, Paris, Presses de Sciences Po.

Liotard P., Terret T., (2005), Sport et genre, vol. 2, Excellence féminine et masculinité hégémonique, Paris, L'Harmattan.

Mardon A., (2010), «Pour une analyse de la transition entre enfance et adolescence », Agora débats/ jeunesses, $n^{\circ} 54$, pp. 13-26.

Massot M.-H., Zaffran J., (2007), «Auto-mobilite urbaine des adolescents franciliens », Espace, populations, sociétés, $\mathrm{n}^{\circ} 2-3$, pp. 227-241.

Mennesson C., Neyrand G., (2010), «La socialisation des filles et des garçons dans les pratiques culturelles et sportives », in S. Octobre (éd.), Enfance et culture. Transmission, appropriation et représentation, Deps/ ministère de la Culture et de la Communication pp. 147-166.

Naves M.-C., Octobre S., (2014), « Lutter contre les stéréotypes filles-garçons. Un enjeu d'égalité et de mixité dès l'enfance », in M.-C. Naves, V. Wisnia-Weil (éds.), Inégalités et différences filles-garçons dans les pratiques sportives et culturelles des enfants et des adolescents, Paris, Commissariat général à la stratégie et à la prospective, pp. 139-169.

Oppenchaim N., (2012), Mobilité quotidienne, socialisation et ségrégation: une analyse à partir des manières d'habiter des adolescents de zones urbaines sensibles, thèse de doctorat, sous la direction de F. Godard, université Paris-Est, laboratoire Ville, Mobilité Transport (LVMT)

Papon F., (1997), « Les modes oubliés : marche, bicyclette, cyclomoteur, motocyclette », Recherche Transports Sécurité, n56, pp. 61-75.

Pfefferkorn R., (2007), Inégalités et rapports sociaux. Rapports de classes, rapports de sexes, Paris, La Dispute.

Raibaud Y., (2014), "Une ville faite pour les garçons », Le Journal du CNRS, [en ligne] https://lejournal.cnrs.fr/ billets/une-ville-faite-pour-les-garcons 


\section{Biographie}

Ripoll, (2006), « Réflexions sur les rapports entre marquage et appropriation de l'espace », in T. Bulot et V. Veschambre (dir.), Mots, traces, marques: dimension spatiale et linguistique de la mémoire urbaine, Paris, L’Harmattan, pp. 15-36.

Rivière C., (2014), Ce que tous les parents disent ? Approche compréhensive de l'encadrement parental des pratiques urbaines des enfants en contexte de mixité sociale (Paris-Milan), thèse de doctorat, Institut d'études politiques de Paris.

Robeyns I., (2000), « An unworkable idea or a promising alternative?: Sen's capability approach re-examined », Econometrics.

Sen A., (1988), «The concept of development », Handbook of development economics, $n^{\circ}$ 1, pp. 9-26.

Soulet M.-H., (2009), « Changer de vie, devenir autre: essai de formalisation des processus engagés », in M. Bessin, C. Bidart, M. Grossetti (éds.), Bifurcations. Les sciences sociales face aux ruptures et à l'événement, Paris, La Découverte, pp. 273-288.

Vigneron C., (2004), La construction des inégalités de réussite en EPS au bac entre filles et garçons, université de Bourgogne, UFR Sciences humaines, Iredu.

Zaffran J., (2016), «Bouger pour grandir. Défection et mobilité des adolescents », Les Annales de la recherche urbaine, $\mathrm{n}^{\circ} 111$, pp. 68-77.

Zaidman C., (2007), "Jeux de filles, jeux de garçons », Les cahiers du CEDREF, [en ligne] https://cedref.revues. org/461

Zolesio E., (2010), « Dispositions "féminines", dispositions "masculines"», Interrogations, pp. 117-126.
DAVID SAYAGH est doctorant à I'Institut français des sciences et technologies des transports des aménagements et des réseaux (Ifsttar) dans le laboratoire du Dest (Dynamiques économiques et sociales des transports). Titulaire de deux masters aux contenus aussi éloignés que complémentaires - Prévention et éducation pour la santé par les activités physiques et sportives, et Urbanisme et aménagement mention Transports et mobilité il s'intéresse aux dimensions structurées et structurantes de la mobilité à vélo, et aux processus de (re)production des rapports sociaux de sexe.

david.sayagh@ifsttar.fr 